\title{
Kinetics and Equilibrium of Crude Oil Sorption from Aqueous Solution Using Borassus Aeothopum Coir
}

\author{
Arinze-Nwosu U. L ${ }^{1} \quad$ Ajiwe V. I. E ${ }^{2} \quad$ Okoye P. A. C ${ }^{2} \quad$ Nwadiogbu J. O. ${ }^{3}$ \\ 1.Raw Materials Research \& Development Council, Enugu State Co-ordinating Office Enugu \\ 2.Department of Pure and Industrial Chemistry, Nnamdi Azikiwe University Awka \\ 3.Department of Chemistry, Chukwuemeka Odumegwu Ojukwu University Uli, Anambra State Nigeria
}

\begin{abstract}
In this study, Borassus aethopum coir (BAC) which is an abundant, cheap, readily available and biodegradable agricultural waste was modified by acetylation using acetic anhydride and NBS catalyst at room temperature. This was to increase their hydrophobic properties and improve their sorption capacity in aqueous environment. The crude oil sorption kinetic data were fitted by intra-particle diffusion and pseudo-first order for raw BAC but the pseudo-second order kinetic model best fits crude oil sorption data for acetylated BAC. The Langmuir and Freundlich models were used to elucidate the sorption behaviour of crude oil with raw and acetylated BAC. Freundlich isotherm gave the best fit for the equilibrium crude oil sorption data for acetylated BAC and raw BAC. Fourier transform infrared and scanning electron microscope analyses showed clear evidence of successful acetylation. The results presented and discussed in the study suggests that acetylated BAC (ABAC) is more suitable for crude oil sorption than raw BAC, therefore, possesses more potential for application and further development in treatment of oil spillage.
\end{abstract}

Keywords: acetylated borassus aethopum coir, kinetics, crude oil sorption, sorbents, sorption capacity.

DOI: $10.7176 / \mathrm{CMR} / 11-2-02$

\section{Introduction}

Niger Delta is the oil producing region of the most populous black African nation Nigeria. Incessant oil spillage in this region is majorly due to pipeline vandalization, oil bunkering, oil exploration and transportation which has devastated arable farmlands, water bodies, lives and properties Oil spills over the oceans and seas requires prompt attention due to their environmental and economic impacts [1]. When oil is spilled in water or on land, the physical and chemical properties of oil changes progressively. The spilled oil contributes an undesirable taste and odour to drinking water and causes severe environmental damages [2]. Oil spill on river and sea envelopes the water surface and consequently shields the diffusion of sunlight that enhances photosynthesis. This adversely affects the aquatic lives that rely mostly on phyto-planktons and seaweeds for existence [3]. The negative impact of oil spill is not peculiar to aquatic lives alone but can also affect the soil if discharged on land and the coastline. This drastically renders the soil unfertile which will result to shortage of agricultural produce supply. Sorbent materials have high attraction for oil. They remove oil by two mechanisms adsorption or absorption. Adsorption involves the adherence of oil to the sorbent material which is dependent upon the viscousity of the oil. The more viscous the oil, the thicker the layer that will adhere to a given sorbent material. Absorption on the other hand relies on capillary action, where oil fills the pores within the material and moves upward into the material due to capillary force [4]. Adsorption is a promising and cost effective method to reduce the environmental problems of oil spill and clean up these types of pollution.

Sustainable oil clean up approach is still a huge and challenging task due to high cost and environmental impact. Strategies and efforts for cleanup activities depend upon various factors such as water waves, weather, currents and response speed. These factors create limitations which poses challenges in recent years. The need for eco-friendly and cost effective natural sorbents cannot be over emphasized in recent times.

Agricultural sorbents are cheap, efficient, environmentally friendly, readily available and easy to deploy.. Several natural organic sorbents have been studied for the removal of oil spill e.g. raw sugar cane bagasse $[5,6$, 7 ], barley straw [8], peat based sorbents [9], banana trunk fibers [10], acetylated corn cob [11], acetylated Dacryodes edulis leaf [12]. These materials are oleophilic because of their waxy nature. They become light weight when dried which improves their buoyancy in water. Natural sorbents and a wide variety of natural organic products such as rice straw, corn corb, peat moss, wood, cotton, milkweed floss, kapok, kenaf and wool fibres are presently attracting attention for development as sorbents for oil spill cleanup applications [1-5]. This is due to their relatively high sorption capacity, biodegradability and cost-effectiveness in comparison to the synthetic polymeric fibres that are normally used.

The effectiveness of the sorbents in saturated aquatic environment would be enhanced if the density of the hydroxyl functional groups is decreased [13]. The hydroxyl functionality of these fibers can be reduced by chemical modification such as acetylation, methylation, cyanoethylation, acrylation, acylation e.t.c [14].

Huge amounts of agricultural wastes are generated in Nigeria annually but only a fraction of these materials are reused because they are thereafter used majorly as domestic sources of fuel for cooking. Borassus aeothopum 
coir (BAC) is one of many types of natural fibres. It is renewable, sustainable and largely unexploited. Besides, $B A C$ is abundantly available and amenable to chemical treatment to alter its morphological, chemical and physical properties. It is a coarse and soft fibre extracted from the tissues surrounding the seed of the borassus fruit /nut which looks like coconut (Cocos nucifera). The coir is left to rot and waste after removing the nut for consumption.

This research is aimed at investigating the effect of acetylation on the borassus aeothopum coir under mild conditions, and its subsequent trial in oil spill cleanup and to understand the kinetics an equilibrium of acetylation and sorption.

\section{Materials and Methods}

\subsection{Sample Preparation}

BAC was sourced from Nnamdi Azikiwe University Awka in Anambra State Nigeria. Thorough washing was done with water to remove fungus, dirts, other foreign materials and water soluble components. The coir was sun dried for 12 hours and then left to dry at $65^{\circ} \mathrm{C}$ in an oven. They were size reduced and sieved through 20 and 25 British Standard Sieve (BSS Sieves). Reagents and chemicals of analytical grade used were from British Drug House (BDH) and include acetic anhydride, N- Bromosuccinimide (NBS), acetone, ethanol and n-hexane, and were used without further purification.

\subsection{Acetylation of BAC}

The method as described by [2] was adopted for the acetylation of BAC under mild conditions, in the presence of NBS, using acetic anhydride. The amount of substrate and reactant were combined in a ratio of 1:20 ( $\mathrm{g}$ dried sorbent $/ \mathrm{mL}$ acetic anhydride). The reaction temperature, time and amount of catalyst were $30^{\circ} \mathrm{C}, 1-2 \mathrm{hrs}$ and $0-4 \%$ respectively. The mixture of raw sorbents, acetic anhydride and catalyst was placed in a round bottom flask fitted to a condenser. The flask was placed in an oil bath on top of a thermostatic heating device, thereafter, the flask was removed from the bath and the hot reagent was decanted off. The sorbents were thoroughly washed with ethanol and acetone to remove unreacted acetic anhydride and acetic acid as by-products. The new products were dried in an oven at $60^{\circ} \mathrm{C}$ for 16 hours prior to analysis. The degree of acetylation was estimated from the infrared spectra by calculating the ratio $(\mathrm{R})$ between the intensity of the acetyl $\mathrm{C}=\mathrm{O}$ stretching band around $1740-1745 \mathrm{~cm}^{-}$ ${ }^{1}$ and the intensity of the C-O stretching vibrations of cellulose backbone at about $1020-1040 \mathrm{~cm}^{-1}$ as shown below $[20]$.

$$
R=\frac{I_{1740}}{I_{1020}}
$$

\subsection{FTIR and SEM Analysis}

The properties of raw and acetylated samples were characterized using FT-IR Shimadzu 8400s spectrophotometer in the range of 4000-400 $\mathrm{cm}^{-1}$. The topographical and morphological information about the sample was provided by SEM through the high-resolution, three dimensional and high depth-of-field images of the sample surface and near-surface. This revealed spatial variations in chemical compositions of the samples and their porous natures.

\subsection{Treatment protocol}

The method as described by [21] was employed in the sorption of oil from water. A portion (1g) of raw and acetylated samples were placed in a $250 \mathrm{ml}$ beaker containing a mixture of crude oil displaced in $100 \mathrm{ml}$ of water at $26^{\circ} \mathrm{c}$. The samples were left in the mixture for about 3 minutes with little agitation. The sorbent was removed from the beaker using sieve nets. The nets were allowed to drain. The oil loaded sorbents were dried at $60^{\circ} \mathrm{C}$ for 30 minutes and reweighed. The oil sorption capacity was calculated by taking into account the weight of sorbent, weight of sorbent and oil and weight of sieve net.

Oil sorption capacity $(\mathrm{g} / \mathrm{g})=\left(\mathrm{S}_{\underline{\mathrm{st}}}-\mathrm{S}_{\mathrm{o}}\right)$

Where $\mathrm{S}_{\mathrm{o}}$ is the initial mass of the dry sorbent, $\mathrm{S}_{\mathrm{st}}$ is the mass of the sorbent with oil at the end of the sorption test and the $\left(\mathrm{S}_{\mathrm{st}}-\mathrm{S}_{\mathrm{o}}\right)$ quantity is the net oil sorbed (all of the masses were measured in grams).

The amount of crude oil adsorbed $\left(\mathrm{q}_{\mathrm{e}}\right)$ in $\mathrm{mg} / \mathrm{g}$ was determined using the following mass balance equation:

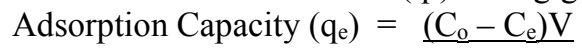

$\mathrm{m}$

where Co is the initial oil concentration in $\mathrm{mg} / \mathrm{L}, \mathrm{Ce}$ is the equilibrium oil concentration $\mathrm{mg} / \mathrm{L}, \mathrm{V}$ is the volume of solution used in litres and $\mathrm{m}$ is the weight of the sorbent material in $\mathrm{g}$. 


\section{Results and discussion}

\subsection{Scanning electron microscope (SEM) analysis}

The SEM micrographs for the raw and acetylated BAC are presented in figs $1 \mathrm{a}$ and $\mathrm{b}$ below. As can be seen, the surface of the untreated sample is very smooth with tiny ripples. Comparing the two, it was observed that the surface morphology of the sorbent BAC changed after acetylation. Figure $1 \mathrm{~b}$ showed that the surface of the modified BAC was more ruptured with different degrees of wrinkles and grooves which increased the surface area. There is noticeable increase in the number of pores in the sorbent after acetylation. Also there is a distinct change in the surface morphology of raw BAC supporting that it was effectively acetylated. These pores can make oil entrance into the internal parts of the material easier and helpful in the sorption process. It is believed that high percentage of oil can be trapped into the porous interior of the sample by capillary mechanism. The macro porous interior fiber enhances the capillary action and the diffusion of oil through the fiber and improves the sorption properties of borassus coir.

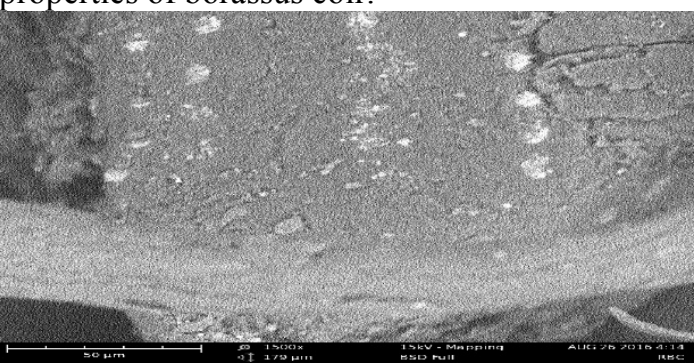

Fig 1a: SEM micrograph for raw BAC

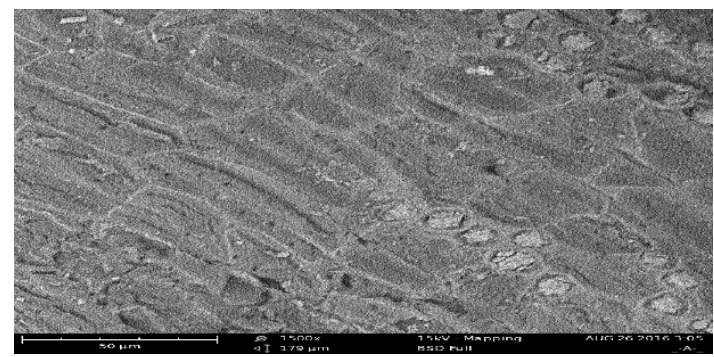

Fig 1b: SEM micrograph for modified BAC

\subsection{Fourier transform infra-red (FTIR) spectra analysis}

Fig $2 a$ and b present infrared spectra of acetylated BAC and raw BAC respectively. The following peak intensities $3994,3909,3749$ and $3407 \mathrm{~cm}^{-1}$ as reflected on the IR spectra of acetic anhydride treated samples are characteristic absorption of bonded - $\mathrm{OH}$ group stretching vibrations in cellulose and hemicelluloses. The intensities increased which is a good evidence of acetylation $[13,31,32]$. Before acetylation, absorption peak was seen at $1649 \mathrm{~cm}^{-1} \mathrm{but}$ this signal shifted to $1643 \mathrm{~cm}^{-1}$ and suggests the presence of hemicellulose [2]. The bond is characteristic absorption of carbonyl $(\mathrm{C}=\mathrm{O}$ ester $)$ stretching vibration of acetate group in cellulose and uronic ester in hemicelluloses. This band showed evidence of acetylation [32, Adebajo and Frost, 2004b). The increase in band absorption from 1140$1142 \mathrm{~cm}^{-1}$ is assigned to $\mathrm{C}=\mathrm{O}$ stretching vibrations in acetyl group (lignin) [13,31]. Fig $2 \mathrm{a}$ and $2 \mathrm{~b}$ show remarkable change in intensity strongly supporting efficient acetylation of BAC. Earlier findings reported for corn cob [2], cotton [34], sugarcane bagasse [6,35], dacryodes edulis leaf [12] are in excellent agreement with presented results that change in absorption intensity suggests effective acetylation. The implication is that some acetyl functional groups (from acetic anhydride) effectively attached to the BAC by replacing the hydroxyl groups. The absence of the band at $1840-1760 \mathrm{~cm}^{-1}$ in all the treated samples showed that the acetylated samples were free of unreacted acetic anhydride $[33,36]$. The absence of the peak at $1700 \mathrm{~cm}^{-1}$ in all the treated samples also indicated that the acetylated products are free of acetic acid by-product.

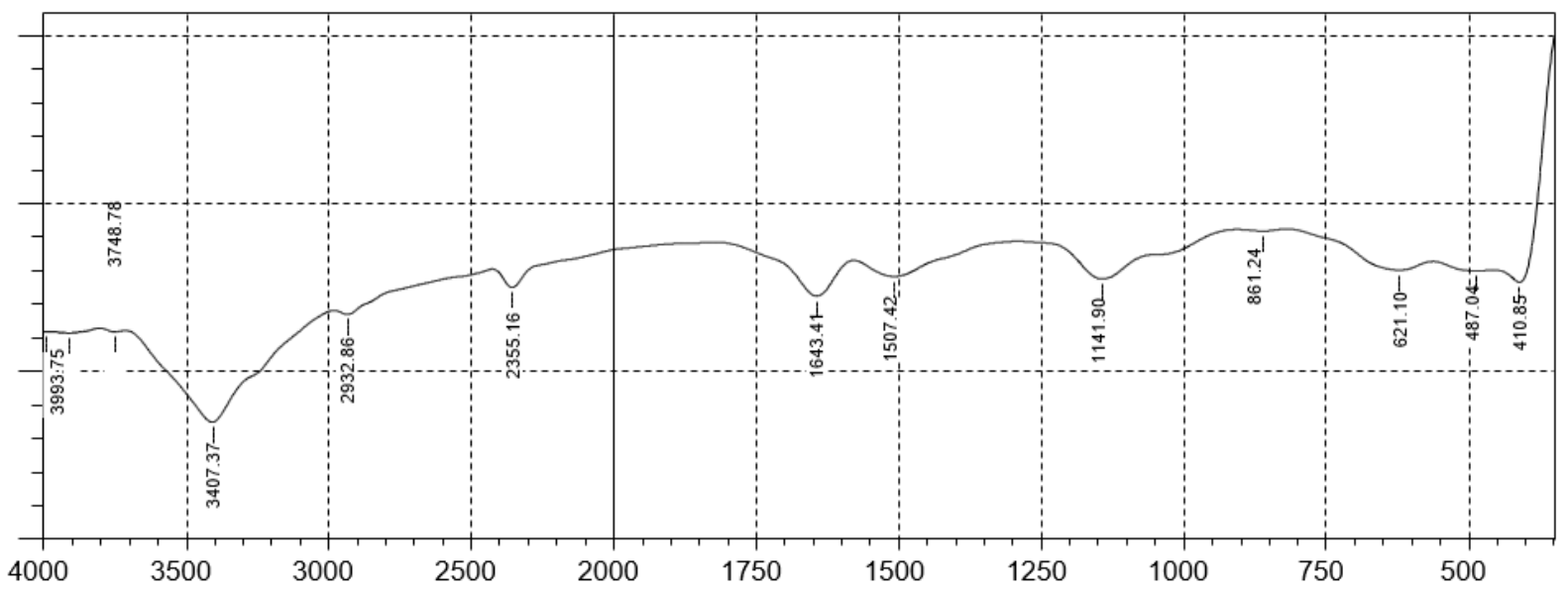

Fig 2a.FTIR Spectra of Acetylated Borassus aeothopum coir (ABAC) 


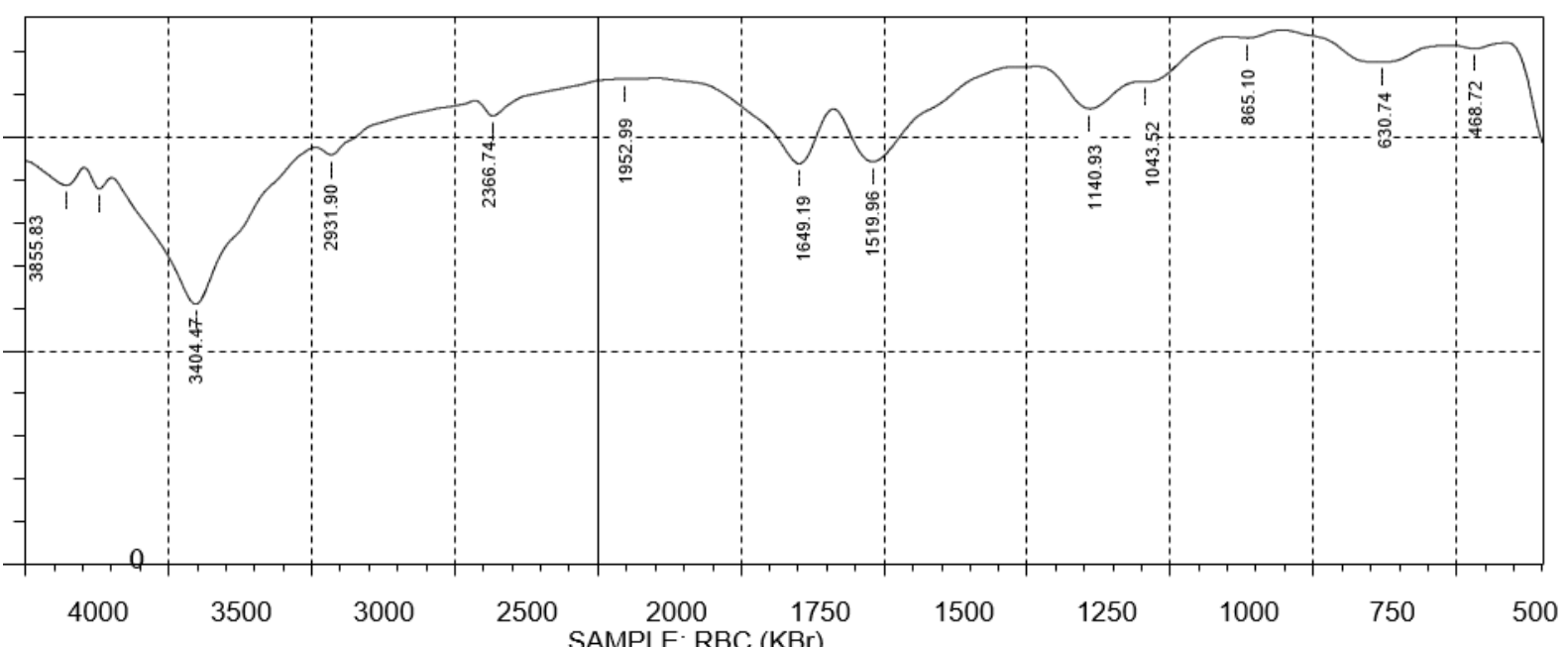

Fig 2b: FTIR spectra of raw BAC

\subsection{Effect of time on crude oil sorption onto BAC}

From figure 3, the results of oil sorption capacity as expected increased with increase in sorption time from 1 to 15 minutes. 15 minutes showed the maximum oil sorption capacity of $9.92 \mathrm{~g} / \mathrm{g}$ for $\mathrm{ABAC}$ and $4.97 \mathrm{~g} / \mathrm{g}$ for raw BAC. This is consistent with findings elsewhere [37]. The crude oil sorption capacity values for the acetylated sample were always higher than those of the raw samples. This might be due to initial adsorption unto the surface of the material and subsequent penetration into the inner microscopic voids [38]. The results further showed the fast and stable nature of the process as only a slight difference was observed between the initial and final contact time. This result is consistent with findings by other researchers $[5,11,19]$. The results as can be seen from Fig 4.57 showed that acetylated BAC absorbed higher amount of oil when compared to the raw BAC. Considering that oleophilicity is a measure of enhanced non-aqueous sorption property, figure 3 demonstrates that $\mathrm{ABAC}$ is more oleophilic than raw BAC. Explanation for this is due to the decrease in hydroxyl functionality of raw BAC by acetylation as ascertained elsewhere [13].

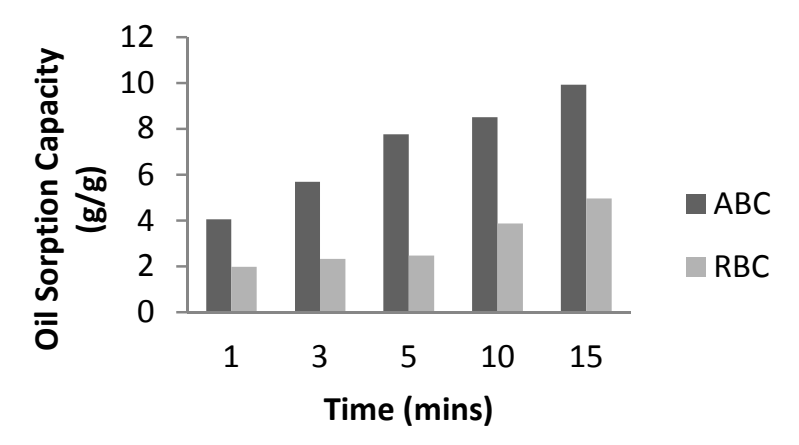

Fig 3 Effect of time on Oil Sorption Capacity of Acetylated and Raw BAC

\subsection{Kinetics of BAC acetylation}

The kinetics for the acetylation of borassus coir was studied by fitting obtained data in rate curves of first order, second order, pseudo-second-order, intra-particle and liquid film diffusion models. 
Table 4: Summary of Kinetics from the Plots of BAC Acetylation

\begin{tabular}{ll}
\hline Kinetic models & Values \\
\hline$\Theta_{0 \text { exp }}$ & 0.94 \\
First order & 1.24 \\
$\Theta_{0 \text { theo }}$ & 0.398 \\
$\mathrm{R}^{2}$ & $4 \times 10^{-4}$ \\
$\mathrm{~K}_{1}$ & \\
Second order & 0.807 \\
$\Theta_{\text {otheo }}$ & 0.4137 \\
$\mathrm{R}^{2}$ & $-5 \times 10^{-4}$ \\
$\mathrm{~K}_{1}$ & \\
Pseudo- second-order & 0.907 \\
$\Theta_{0 \text { theo }}$ & 0.994 \\
$\mathrm{R}^{2}$ & 1.1 \\
$\mathrm{~K}_{1}$ & \\
Intra-particle diffusion $_{\mathrm{K}}$ & $8.5 \times 10^{-3}$ \\
$\mathrm{R}_{\mathrm{d}}^{2}$ & 0.4178 \\
$\mathrm{C}$ & 0.7658 \\
Liquid film diffusion & \\
$\mathrm{K}_{\mathrm{fd}}$ ( meq $\mathrm{g}^{-1} \mathrm{~s}^{1 / 2}$ ) & -0.0057 \\
$\mathrm{R}^{2}$ & 0.2098. \\
\hline
\end{tabular}

From Table 4, the very low values for the regression $\left(\mathrm{R}^{2}\right)$ for first and second order expression 0.398 and 0.4137 respectively. Coefficient of regression $\left(R^{2}\right)$ within $0.43 \leq R^{2} \leq 0.83$ are considered high and moderate [39] therefore the $\mathrm{R}^{2}$ value for first and second order could be said to be very low. This suggests that it is inappropriate to use this kinetics to represent the acetylation of borassus coir. It was also observed that the first and second order produced theoretical values of 1.24 and 0.807 while the experimental value was 0.94 . The pseudo second order gave a very high $\mathrm{R}^{2}$ value of 0.994 which was close to unity indicating that the pseudo second order kinetic model produced a better fit to the data and also a theoretical value of 0.907 which is closer to the experimental value of 0.94 suggesting that it is the best kinetic model to represent the acetylation of borassus coir.

High value of $\mathrm{R}^{2}$ obtained at room temperature pseudo second order kinetic plots also suggests that acetylation of BAC is energy efficient since physical and chemical reaction between the modifier (acetic anhydride and NBS catalyst) and BAC can take place at room temperature. It also implies that high temperature is not favourable for acetylation. Intra-particle diffusion model was also used to further study the mechanism of acetylation. The $\mathrm{R}^{2}$ obtained was low (0.4178). It was expected that the plot of $\mathrm{q}_{\mathrm{t}}$ versus $\mathrm{t}^{1 / 2}$ would give a linear relationship when intra-particle diffusion is involved in the acetylation process and that it would be the controlling mechanism if the line passed through the origin $[40,41]$. However for the case where the plots did not pass through the origin, the reason has been suggested that the intra-particle diffusion was not the only mechanism involved in the acetylation process due to some degree of boundary layer control. $[2,42]$. C is a constant that gives an idea about the thickness of the boundary layer. The larger the value of $\mathrm{C}$, the greater the boundary layer effect

The linearity of the plot of $\ln (1-F)$ as a function of time $t$ for the liquid film diffusion model indicated the high applicability of this model [11], though this did not pass through the origin. Furthermore, the liquid film mechanism produced a poor $\mathrm{R}^{2}(0.2098)$ while the intra-particle diffusion model gave a better $\mathrm{R}^{2}$ value $(0.4178)$. The results of the kinetic studies revealed that, acetylation of borassus coir is by surface reaction on BAC. This is in agreement with results elsewhere [40].

\subsection{Crude oil sorption profile}

3.6.1 Kinetic studies

A good understanding of the kinetics of crude oil sorption onto BAC and ABAC is imperative for practical application. Particularly, the industrial applications can be feasible if some kinetic parameters are obtained for uses in process designs and scale up procedures [43]. The sorption kinetic models were applied to the experimental data to analyze the rate and mechanism of crude oil sorption of $\mathrm{BAC}$ and $\mathrm{ABAC}$. The kinetic models applied to this study are the pseudo first-order, pseudo second-order and intra-particle diffusion models.

The pseudo-first order kinetic model is expresses as [44]

$\ln (q e-q t)=\ln q e-k_{l} t$

where $q t$ and $q e$ are the amount of crude oil sorbed at time $\mathrm{t}(\mathrm{s})$ and at equilibrium, respectively in $\mathrm{mg} / \mathrm{g}$. $k_{l}$ is the pseudo-first order rate constant $\left(\mathrm{s}^{-1}\right)$. The slope and intercept of the plots of $\ln (q e-q t)$ as a function of $t$ were used to determine the rate constant and $q e$, and the values are listed in Table 4.

Similarly, the pseudo-second order kinetic model can be expressed as [45]: 


$t=\frac{1}{k_{2} q_{e}^{2}}+\frac{t}{q e}$

where $k_{2}$ is the rate constant of the pseudo-second order expression (g/mgs-1). The $q_{e}$ and $k_{2}$ values were calculated from the slope and intercept of the linear plot of $t / q t$ as a function of the time $(\mathrm{t})$, and the values are listed in Table 4. A comparison of the kinetic parameters of crude oil sorption on BAC and ABAC is presented in Table 4 below. From the Table 4, it could be seen that the coefficient of regression $\left(\mathrm{R}^{2}\right)$ for crude oil sorption unto $\mathrm{ABAC}$ was 0.9207 for the pseudo-first order, 0.9867 for the pseudo-second order and 0.9517 for intra-particle diffusion. Pseudo-second order gave the best fit for crude oil sorption of $\mathrm{ABC}$ because it gave the highest $\mathrm{R}^{2}$ value (0.9867) which is close to unity. For raw BAC, $\mathrm{R}^{2}$ values are lower for both the pseudo-first and pseudo-second orders at 0.9085 and 0.8871 respectively when compared with that of ABAC. However pseudo- first order gave the best fit with $\mathrm{R}^{2}$ value of 0.9085 . This can be attributed to the hydrophobic nature of the modified sorbent which gave it a higher $\mathrm{R}^{2}$ [46].

Table 4 Summary of Kinetics of Crude Oil Sorption onto BAC

\begin{tabular}{lll}
\hline Type & ABAC & BAC \\
\hline Qe expected & 9915 & 4970 \\
Pseudo first Order & 6299 & \\
$q_{e}$ calc & 0.9207 & 4003 \\
$\mathrm{R}^{2}$ & 0.1605 & 0.9085 \\
$\mathrm{~K}_{1}$ & & 0.1392 \\
Pseudo Second Order & 11,111 & \\
$\mathrm{q}_{\mathrm{e} \text { calc }}$ & 0.9867 & 5000 \\
$\mathrm{R}^{2}$ & 0.00009 & 0.8871 \\
$\mathrm{~K}_{1}$ & & 0.0002 \\
Intra Particle Diffusion & 1989.6 & \\
$\mathrm{~K}_{\mathrm{d}}$ & 2411.8 & 1079 \\
$\mathrm{C}$ & 0.9517 & 517.11 \\
$\mathrm{R}^{2}$ & & 0.9289 \\
\hline
\end{tabular}

The intra-particle diffusion model can be expressed as ( )

$q t=k_{d} \cdot t^{1 / 2}+I$

Where $k_{d}$ is the intra-particle rate constant $\left(\mathrm{mg} / \mathrm{g} \mathrm{s}^{1 / 2}\right)$ and $I$ is the intercept. The intercept of the plot is shown in Table 4 and indicated the presence of a boundary layer effect. When the intercept is larger, surface sorption contributes more to the rate determining step. The $k_{d}$ constant was obtained from the slope of the plot of $q t$ as a function of $t^{1 / 2}$. Intra-particle diffusion becomes the rate determining step when the plot is linear and passes through the origin $[11,44]$. Based on the result presented above, a high regression co-efficient value of 0.9517 and 0.9289 were obtained for ABAC and raw BAC respectively with a non-zero intercept. The deviation from the origin could be attributed to the difference in mass transfer between the initial and final stages of the sorption of crude oil onto ABAC and raw BAC. Also the presence of boundary layer effect $(I)$ confirmed the existence of surface sorption, implying that an intra-particle diffusion mechanism was not the only rate-limiting step.

\subsubsection{Equilibrium studies}

Adsorption isotherm is important to describe the interactions between the solute and the adsorbent. In this study, Langmuir and Freundlich isotherms were tested to analyze the equilibrium data for borassus coir and the results are shown in Table 5.

The Langmuir isotherm was used to describe the adsorption phenomena and is based on the assumption that adsorption occurs uniformly on the active sites of the sorbent. In addition, once an adsorbate occupies a site, no further sorption can occur at that site. The linear form of the Langmuir model [47] can be expressed as:

$\frac{\mathrm{Ce}}{q_{e}}=\frac{1}{q_{o} b}+\frac{\mathrm{Ce}}{q_{o}}$

where $q_{\mathrm{o}}$ is the equilibrium monolayer adsorption capacity of the adsorbent $(\mathrm{mg} / \mathrm{g}), b$ is the Langmuir adsorption constant $(\mathrm{L} / \mathrm{mg})$ related to the energy of adsorption, which quantitatively reflects the affinity between the adsorbent and the adsorbate, and $q_{\mathrm{o}}$ is the maximum monolayer adsorption capacity of the adsorbent $(\mathrm{mg} / \mathrm{g})$. The $q_{\mathrm{o}}$ and $b$ constants can be determined from a linear plot of $C_{\mathrm{e}} / q_{\mathrm{e}}$ as a function of $C_{\mathrm{e}}$. As shown in Table 5, the plot enabled the calculations of Langmuir constants $(b=0.0000341$ for ABAC and 0.0000370 for BAC) and Langmuir theoretical saturation capacity $(\mathrm{qo}=3333.33)$ from values of intercept and slope respectively. The coefficient of determination value $\mathrm{R}^{2}$ is 0.8233 and 0.8415 . These values are not high enough therefore not a very good prediction of the equilibrium data. There is a Langmuir dimensionless constant $\left(R_{L}\right)$, also known as separation factor, known to predict biosorption efficiency which is defined by the following relationship [47].

$\mathrm{R}_{\mathrm{L}} \quad=\frac{1}{1+b} \mathrm{C}_{\mathrm{o}}$

where $C o$ is the initial concentration of crude oil in $\mathrm{mg} / \mathrm{L}$. The separation factor provides information regarding 
the nature of the adsorption process. The adsorption can be considered irreversible $(R \mathrm{~L}=0)$, favourable $(0<R \mathrm{~L}<$ $1)$, linear $(R \mathrm{~L}=1)$ or unfavourable $(R \mathrm{~L}>1)$. The $R \mathrm{~L}$ obtained from this study was 0.0001 , indicating favourable sorption of crude oil on acetylated borassus coir. The Freundlich isotherm model has been applied to non-ideal sorption on heterogeneous surfaces, and the linear form of this equation can be expressed as [44].

$\ln q e=\ln k_{f}+\left(\begin{array}{l}1 \\ n\end{array}\right) \ln C_{e} \quad$ [9]

where $k \mathrm{f}(\mathrm{mg} / \mathrm{g})(\mathrm{mg} / \mathrm{L}) 1 / n$ and $1 / n$ are the Freundlich adsorption capacity and the intensity of the adsorbents, respectively. The constants were determined from the intercept and slope of the linear plot of lnqe as a function of $\ln C$ e. As shown in Table 5, the value of the coefficient of regression $\left(R^{2}\right)$ was 0.9813 , which is higher than that obtained for the Langmuir model at 0.8233. This result indicates that the Freundlich model is suitable for describing the sorption equilibrium of crude oil on acetylated BAC, which is in agreement with other researchers results $[11,19]$. Moreover, the equilibrium constant value predicted by Freundlich isotherm (KF) for both $\mathrm{ABAC}$ and $\mathrm{BAC}$ has value higher than unity. This suggests a more favourable crude oil sorption for ABAC because of it's higher value of 57.32 .

Table 5: Comparison of Langmuir and Freundlich Isotherm Constants for BAC.

\begin{tabular}{lll}
\hline Isotherm Model & ABAC & BAC \\
\hline Langmuir & & \\
$\mathrm{q}_{\mathrm{o}}$ & 3333.33 & 3333.33 \\
$\mathrm{~B}$ & 0.0000341 & 0.0000370 \\
$\mathrm{R}_{\mathrm{L}}$ & 0.370 & 0.395 \\
$\mathrm{R}^{2}$ & 0.8233 & 0.8415 \\
Freundlich & & \\
$\mathrm{k}_{\mathrm{f}}$ & 57.32 & 9.165 \\
$1 / \mathrm{n}$ & 0.663 & 0.8488 \\
$\mathrm{R}^{2}$ & 0.9813 & 0.8921 \\
\hline
\end{tabular}

\section{Conclusion}

The results of acetylation revealed successful acetylation of BAC as proved by the FTIR spectra. The acetylation was effective and can be achieved under mild conditions and therefore environmentally safe and economically viable.

This study indicates that BAC which is an agricultural waste can be deployed as a sorbent for oil spill treatment. Crude oil sorption kinetic data were best fitted by intra-particle diffusion and pseudo-first order for raw BAC. But the pseudo-second order kinetic model best fits crude oil sorption data for ABAC. The Langmuir and Freundlich models have been used to elucidate the sorption behaviour of crude oil with raw and acetylated BAC. Freundlich isotherm gave the best fit for the equilibrium crude oil sorption data for ABAC and raw BAC.

The crude oil sorption capacity of raw BAC was enhanced while the water absorption capacity was reduced for the modified sample ABAC. Therefore, ABAC can be deployed as an effective sorbent for oil spillage treatment. BAC which is an abundantly available agricultural waste, cheap, biodegradable and economical to modify by acetylation, the acetylated BAC is therefore highly recommended for non-aqueous sorption processes

\section{References}

1. T. R. Annunciado, T. H. D.Sydenstricker, and Amico, S. C. Experimental Investigation of various vegetable fibers as sorbent materials for oil spills. Marine Pollution Bulletin, 50(11): (2005). 1340-1346.

2. J. O. Nwadiogbu P. A. C. Okoye, V. I. E. Ajiwe and N. J. Nnaji. Hydrophobic treatment of corncob by acetylation: Kinetics and thermodynamic studies. Journal of Environmental Chemical Engineering. Vol:2, (2014). pp $1699-1704$

3. J. Idris,, G. D Eyu, C. Zamani, E. Ahmad, Oil spills and sustainable clean up approach. Australian J. of basic and Applied Sc. 7 (14), (2013). 272-280.

4. C. P. Karan, R. S. Rengasamy, D. Das, Oil spill cleanup by structured fibre assembly, Indian Journal of Fibre Text. Res. 26 (2011) 190-191.

5. M. Hussein, A. A. Amer and I. I. Sawsan. Oil spill sorption using carbonized pith bagasse: Trial for practical application. International Journal of Environmental Science and Technology, 5(2): (2008), 233-242.

6. P. C. Brandao and T. C. Souza. Removal of petroleum hydrocarbons from aqueous solution using sugarcane bagasse as adsorbent. Journal of Hazardous Materials, Vol 175, (2010).

7. A. A. Said, A. G. Ludwick, H. A Aglan, Usefulness of raw bagasse for oil absorption: A comparison of raw and acylated bagasse and their components, Bioresource Technology. 100 (2009), 2219-2222.

8. M. Hussein, A. A. Amer, A. El-Maghraby, A. Taha, Availability of barley straw application on oil spill cleanup, Int. J. Environ. Sci. Technol. 6 (2009) 123-130.

9. C. Cojocaru, M. Macovearu, and I. Cretescu. Peat-based sorbents for the removal of oil spills from water surface: Application of artificial neural network marketing. Colloids and Surfaces A: Physicochemical and 
Engineering Aspects. Vol. 384 (1-3), 2011.

10. K. Sathasivam, M. Haris. Adsorption Kinetics and capacity of fatty-acid modified banana trunk fibers for oil in water. Water, Air and Soil Pollution, An international Journal of Environmental Pollution. Vol. 213 (1-4) 413-423 2010.

11. J. O. Nwadiogbu P. A. C. Okoye, V. I. E. Ajiwe, Removal of crude oil from aqueous medium by sorption on hydrophobic corncobs: Equilibrium and Kinetic studies. Journal of Taibah University of Science. Vol: 10, (2016), $56-63$.

12. J. N. Nnaji, T. U. Onuegbu, O. Edokwe, G. C. Eze, and A. P. Ngwu. An approach for the reuse of Dacryodes edulis leaf: Characterization, acetylation and crude oil sorption studies. Journal of Environmental Chemical Engineering. Vol 4: (2016). pp 3205-3216.

13. R. Bordilau and C. A. Teaca. Fourier Transform Infrared Spectroscopy and thermal analysis of ligno cellulose fillers treated with organic anhydrides. Romanian Journal of Physics, Bucharest, 54 (1-2): (2009). 93-104.

14. G. A. Breitenbeck, B. Grace, M. Holiday, M. Louisiana applied oil spill research and development program, OSTRADP Technical Report series 96-001 (1997).

15. American Standards for Testing and Materials (ASTM) D442-84: Standard Test Method for Direct Moisture Content of Wood and Wood based Materials. ASTM, 04-09: (1994), 514-518.

16. M. O. Adebajo, and R. L. Frost. Acetylation of raw cotton for oil spill cleanup application: An FTIR and 13C MAS NMR spectroscopic investigation. Spectrochimica Acta, Part A: Molecular and Biomolecular Spectroscopy, 60 (10): (2004). 2315-2321.

17. S. S. Banerjee, M. V. Joshi and R. V. Jayaram. Treatment of oil spills using organo-fly ash. Desalination, (2006). 195:32-39.

18. A. Yakubu, G. Olatunji, O. Sunday and A. Olubunmi. Ketene acetylated wood cellulose for industrial application in wood base and polymer industry. Environ. Sci. Technol 5: 168- 176. 2013

19. B. Mohebby, F Ilbeigi, S. Kazami-Najafi (2008). Influence of hydrothermal modification of fibres on some physical and mechanical properties of medium density fibreboard (MDF). Holz Roh-Werkst 66(3): 213-218.

20. C. Senanurakwarkul, T. Chavarmakul, The study of factors influencing the decision to select the use of oil sorbent in Thailand. Int. J. Res. Mgt. Technol. 2 (6) (2012) 955-963.

21. G. Ramadoss, K. Muthukumar, Mechanistic study on ultrasound assisted pretreatment of sugarcane bagasse using metal salt with hydrogen peroxide for bioethanol production, Uitrason, Sonochem, 28 (2016) 207-217.

22. Y. Sun, J. Cheng, Hydrolysis of lignocellulosic materials for ethanol production: A review. Bioresource Technology, 83: (2002). 1-11.

23. J. Roja, S. Moren, A. Lopez, Assessment of the water sorption properties of several microcrystalline celluloses. J. Pharm. Sci. Res. 3 (7) (2011) 1302-1309.

24. A. A. Amer, A. El-Maghrahy, G. F. Malash, T. A. Nahla, Extensive characterization of raw barley straw and study of the effect of steam pretreatment. Journal of Applied Science Resources, 3 (1), (2007). 1336 -1342.

25. N. M. Dowine, R> W. Heath, Basic statistical methods, fourth ed. Harper and Row, New York, (1974).

26. C. A. S. Hill, D. Jones, G. Strickland, N. S. Cetin, Kinetic and mechanistic aspects of the acetylation of wood with acetic anhydride, Holzforschung 52 (1998) 623-629.

27. J. C. Igwe, A. A. Abia, A bioseparation process for removing heavy metals from wastewater using biosorbents, African J. of Biotech, 5 (12) (2006), 1167-1179

28. E. Bulut, M. Ozacar, I. A. Sengil, Adsorption of malachite green on bentonite: equilibrium and kinetic studies and process design. Microporous Mesoporous materials, 115 (2008). 234-246.

29. D. Faith, Dye removal by almond shell residues: Studies on biosorption performance and process design, Material Sci. Eng C. 33 (2013) 2821-2826.

30. F. A. Dawodu, K. G. Akpomie, Simultaneous adsorption of $\mathrm{Ni}(11)$ and $\mathrm{Mn}(11)$ ions from aqueous solution on to a Nigerian Kaolinite clay. J. Material Res. Technol, 3 (2) (2014) 129-141.

31. Y. S. Ho, G. Mackay. The Kinetics of sorption of basic dyes from aqueous solution by sphagnum moss peat. Canadian J. of Chem. Eng. 76 (1998) 822-827.

32. J. C. Onwuka, E. B. Agbaji, V. O. Ajibola, F. G. Okibe,.(2016). Kinetics studies of surface modification of lignocellulosic delonix regia.

33. I. Langmuir, The adsorption of gases on plane surfaces of glass mica and platinum. J. Am. Chem. Soc. 40 (1918)1361-1403. 